Las inasistencias de los estudiantes: la escuela seCundaria en Cuestión. Páginas 47-68 en Revista de la Escuela de Ciencias de la Educación, AÑo12, NRO.11, VOL. 1, ENERO A JUNIO de 2016. ISSN 1851-6297. ISSN en Línea 23623349 .

\title{
LAS INASISTENCIAS DE LOS ESTUDIANTES: LA ESCUELA SECUNDARIA EN CUESTIÓN
}

\author{
Andrea Martino* \\ Universidad Nacional de Córdoba. \\ Instituto de Capacitación e Investigaciones de la \\ Unión de Educadores de la Provincia de Córdoba, Argentina. \\ andreagmartino@gmail.com
}

Recibido: 06/03/2016 Aceptado: 27/04/2016

\section{Resumen}

En este artículo (1) se presentan avances de la investigación que se viene realizando sobre el ausentismo de los estudiantes en la escuela secundaria. Se trata de un estudio de caso en una escuela de gestión estatal ubicada en una localidad aledaña a Córdoba Capital.

Iniciamos el texto presentando la problemática de las inasistencias escolares como un analizador de las tensiones entre las definiciones de la política pública que extienden y democratizan el tiempo escolar y las dificultades para hacer que ese tiempo se traduzca en aprendizajes efectivos. Partimos de considerar al ausentismo estudiantil como discontinuidad, interrupción y disminución del tiempo para aprender y que en su producción hay múltiples razones y condiciones, por lo cual es necesario pensar en formas de ausentismo.

El trabajo de campo permite reconocer las voces de aquellos que suelen estar ausentes en las escuelas, y acercarnos a los obstáculos y condiciones que dificultan efectivamente su presencia en la escuela; también a los sentidos que van construyendo en un tiempo que es para aprender, en coincidencia o no, con sus otros tiempos vitales.

Las reflexiones finales invitan a preguntarse y pensar alternativas acerca de las ausencias de los estudiantes desde apuestas más integrales de trabajo con los jóvenes.

* Profesora en Ciencias de la Educación. Universidad Nacional de Córdoba. Licenciada en Ciencias de la Educación. Universidad Nacional de Córdoba. Especialista en la Enseñanza de las Ciencias Sociales, con mención en Formación Ética y Ciudadana. Facultad de Filosofía y Humanidades, Universidad Nacional de Córdoba. Docente Asistente Dedicación Semi en la cátedra "Análisis Institucional de la Educación" de la carrera de grado en Ciencias de la Educación, Universidad Nacional de Córdoba. 
Revista de la Escuela de Ciencias de la Educación, año12, nRo.11, vol. 1, enero a Junio de 2016. Páginas 47-68. ISSN 1851-6297. ISSN EN LIINEA 2362-3349. LAS INASISTENCIAS DE LOS ESTUDIANTES: LA ESCUELA SECUNDARIA EN CUESTIÓN. AndREa MarTino

\title{
Palabras Clave:
}

Inasistencias Escolares - Escuela Secundaria - Forma Escolar - Presencialidad - Tiempo Para Aprender.

\begin{abstract}
In this article progress of a research work studying student absenteeism in secondary schools is presented. It is a case study in a state-run school located in a town near Córdoba city.

We start the text setting out the approach to the issue of non-attendance at school. We consider it as a powerful concept to analyze tensions that arise between public policy definitions adding and democratizing time to school and difficulties to turn that time into effective learning. We assume student absenteeism means discontinuity, interruption and reduction of time to learn. We also believe there are multiple reasons and conditions that foster this problem being therefore necessary to distinguish between different forms of absenteeism.

Research fieldwork highlights the voices of those who are frequently absent from school, leading us to the set of obstacles and conditions that difficult these students' attendance at school. Consequently we are in better conditions to understand the meanings they construct in a time to learn that may match students' vital time or not.

Final reflections are presented as an invitation to ask and think of alternatives towards improving students' attendance at school from a more integrated perspective on work with youngsters.
\end{abstract}

\section{Keys Words:}

School Absences - High School - School Form - Presentiality - Time To Learn.

\section{Introducción}

En las últimas décadas, el Estado ha venido desarrollado políticas tendientes a incrementar los tiempos de escolarización, tanto a través de la extensión de la obligatoriedad a toda la escuela secundaria, como otras medidas más puntuales, como la Jornada Extendida en las escuelas primarias, la incorporación de las salas de tres años al nivel inicial, el aumento a 180 días de clase en el calendario escolar. Esto representa un gran avance en términos políticos y sociales, pues implica el reconocimiento de que para integrarse, en sociedades complejas y desiguales como la nuestra, como ciudadanos plenos, se necesita de más tiempo escolar para la transmisión de los saberes y recursos culturales a los que las nuevas generaciones tienen derecho, en especial las más vulnerables socialmente.

Este incremento en los tiempos de escolarización, se traduce en la vida de niño/as y jóvenes en más tiempo de escuela. Un tiempo que para el caso de la escuela secundaria, ha dejado de ser selectivo para constituirse en obligatorio 
Revista de la Escuela de Ciencias de la Educación, año 12, nRo. 11, vol. 1, enero a junio de 2016. Páginas 47-68. ISSN 1851-6297. ISSN EN LIINEA 2362-3349. LAS INASISTENCIAS DE LOS ESTUDIANTES: LA ESCUELA SECUNDARIA EN CUESTIÓN. AndREa MARTINo

para todo/as, del que el Estado aparece como garante y responsable de hacer cumplir según la Ley Nacional de Educación N² 26206 en sus artículos 2 y 4.

Mientras se producen estos avances, se vienen identificando -sin embargo- problemas ligados al modo en cómo este tiempo escolar se traduce en aprendizajes efectivos. Las elevadas cifras de las inasistencias de algunos estudiantes a la escuela, parecen ponerlo en jaque. Es en esta línea, que la cuestión del ausentismo en la escuela secundaria, es un asunto que merece algunas consideraciones puesto que, entendemos, su complejidad en parte es producto de las razones y condiciones, sociales y subjetivas, que llevan a que un joven falte reiteradamente a la escuela, pero también de las posibilidades y constricciones que actualmente tienen las escuelas, sus agentes y las propias políticas educativas para abordarlas de modos más integrales.

\section{Pensar las ausencias de los estudiantes... una perspectiva posible}

La ausencia de los estudiantes en la escuela, es ausencia de sus cuerpos, pero también es ausencia en un tiempo destinado al aprendizaje y a la posibilidad de experiencias educativas significativas. La irregularidad, la discontinuidad en los aprendizajes, en el seguimiento y profundización de un determinado tema curricular, en las dinámicas grupales que pueden producirse al interior del grupo clase, en los acuerdos e incluso los desacuerdos que se producen cotidianamente durante la jornada escolar, configuran desenganches en los estudiantes respecto a su proceso escolar.

Desde la perspectiva centrada en las trayectorias escolares, Terigi indica dos cosas a propósito del ausentismo, que en este trabajo se recuperan. Por un lado, a diferencia de otras problemáticas, como la sobreedad y la repitencia cuya producción se halla en íntima relación con los rasgos de una gramática escolar graduada, anualizada y por niveles, las inasistencias de los estudiantes en la escuela, se derivan de múltiples razones y motivos que no necesariamente tienen que ver con lo que hace o deja de hacer la escuela. Sin duda, y tal como señala la autora "no hay un único motivo de ausentismo: se falta a las primeras horas de clase del turno nocturno porque se superponen con el horario de trabajo; se está ausente durante un período prolongado como consecuencia de la maternidad, o de trabajos ocasionales; se abandona temporalmente y luego se retoma la escolaridad, en el marco de una experiencia escolar de baja intensidad signada por inasistencias esporádicas pero numerosas y por una relación tensa con la escuela" (2007). De hecho propone hablar de "modos de ausentismo" tratando con ello de reconocer la heterogeneidad de situaciones sociales, escolares y vitales que están en la base de esta problemática (2010).

Por otro lado, Terigi señala que el ausentismo es uno de los problemas aún no resueltos, que signan las trayectorias escolares de muchos adolescentes en la escuela secundaria. Escribe "este asunto enfrenta a las escuelas con 
Revista de la Escuela de Ciencias de la Educación, año 12, nRo. 11, vol. 1, enero a junio de 2016. Páginas 47-68. ISSN 1851-6297. ISSN EN LIINEA 2362-3349. LAS INASISTENCIAS DE LOS ESTUDIANTES: LA ESCUELA SECUNDARIA EN CUESTIÓN. AndREa MARTINo

una problemática central: la dificultad para garantizar los aprendizajes en condiciones que producen una disminución significativa del tiempo de exposición de los estudiantes a la instrucción y una discontinuidad de la experiencia de aprendizaje" (Terigi, 2007).

Interesa pensar el ausentismo de algunos estudiantes como una problemática que tiene que ver con el tiempo escolar: por un lado como disminución del tiempo para aprender, aquel establecido por el Estado como el necesario para apropiarse y aprender los saberes y bienes culturales a los que las nuevas generaciones tienen derecho; y por otro lado, como discontinuidad en ese tiempo para aprender, y en la posibilidad de que se produzcan relaciones significativas con el saber.

En el actual formato escolar, el tiempo para aprender es el tiempo de la presencia de los estudiantes en la escuela. Esto es, los estudiantes deben asistir diariamente a clases durante un tiempo cuya duración es variable (según el nivel educativo, los ciclos y el tipo de institución) el que se organiza internamente (módulos, horas cátedra, recreos) de determinada manera en pos del logro de ciertos fines educativos. Cuando la regularidad en la presencia se interrumpe, ello afecta el tiempo que los alumnos están bajo la exposición de acciones de enseñanza y esto afecta su rendimiento académico, puede deteriorar y empobrecer sus aprendizajes y la producción de sentidos más fértiles con respecto a la escuela.

En un estudio elaborado conjuntamente entre la DINIECE y Unicef sobre las dificultades en las trayectorias escolares de los alumnos en el año 2004, se planteaba el ausentismo y la repitencia como problemáticas asociadas a la sobreedad. "La ausencia prolongada a la institución escolar plantea una serie de discontinuidades en las tareas de enseñanza y consecuentemente en el aprendizaje de los alumnos" se señalaba en el documento (DINIECE-Unicef, 2004).

En un informe elaborado por la Fundación SES (2) se partía de la idea que el ausentismo de los jóvenes a la escuela incide en las trayectorias educativas, en cuanto a los aprendizajes, desempeño escolar, y permanencia. Por otra parte, se constituye en un factor que desencadena en muchos casos situaciones de repitencia no solo por abandonos anuales, sino también por la escasa presentación a las mesas de exámenes de Diciembre y o Marzo (s/f).

En forma reciente, entre los resultados de las pruebas PISA2012, se pone de manifiesto que nuestro país "tiene la tasa más alta de ausentismo estudiantil de todos los países participantes en PISA. Más de la mitad de los alumnos de secundaria evaluados admite haber faltado a clase y llegado tarde. No debe sorprender que ambos comportamientos están negativamente relacionados al desempeño". Y aporta los siguientes datos: "Alrededor de la mitad de los jóvenes argentinos de 15 años llega tarde a la escuela". "Los estudiantes argentinos que llegaron tarde a clase cinco días o más en dos semanas están un grado escolar por detrás de sus pares puntuales" (Gaminián, 2014). 
Revista de la Escuela de Ciencias de la Educación, año 12, nRo. 11, vol. 1, enero a junio de 2016. Páginas 47-68. ISSN 1851-6297. ISSN EN LINEA 2362-3349. LAS INASISTENCIAS DE LOS ESTUDIANTES: LA ESCUELA SECUNDARIA EN CUESTIÓN. AndREa MARTIno

Estas referencias nos indican que estamos ante una problemática que afecta en mayor o menor grado el rendimiento académico de los adolescentes escolarizados, siendo productora de repitencia y por tanto de sobreedad, pues lo que está en juego es el tiempo que dispone la escuela para que sus estudiantes aprendan. Ese tiempo se caracteriza por ser presencial. Ello significa que la presencialidad regula y condiciona las oportunidades de aprendizaje. Sin embargo hoy las formas de estar presentes y ausentes en la escuela por parte de algunos estudiantes pone en tensión estos supuestos y obliga a pensar en alternativas para la discontinuidad. A su vez, en el caso del ausentismo, las razones, motivos y/u obstáculos que llevan a que un joven "decida faltar a la escuela" escapan muchas veces al control y a las posibilidades de acción de los propios agentes escolares, desde los organigramas funcionales instituidos y lo que hacen las escuelas para abordarlo terminan en las fronteras del edificio escolar.

\section{Exploraciones sobre algunas cifras del ausentismo... alcances y límites para mirar esta problemática}

Si bien los avances de la investigación que aquí se presentan remiten a un estudio de caso, interesa traer algunos datos que, por supuesto no son representativos estadísticamente pero sí permiten registrar aunque sea en forma un tanto embrionaria tendencias al respecto (3). Cabe señalar que no existen estadísticas de alcance nacional y provincial sobre el ausentismo, considerado como un indicador que pueda dar cuenta de las dificultades para permanecer y egresar del sistema educativo. Si bien viene siendo una problemática reconocida por muchas escuelas -por lo menos las de gestión estatal- no se han producido aún modalidades específicas que permitan reconocer estadísticamente la magnitud del fenómeno en la escala del sistema educativo, así como generar herramientas de información pertinentes para reconocer e identificar las causas o razones que lo generan.

Así por ejemplo, en el año 2008, en una institución de gestión estatal en Córdoba Capital ubicada en un contexto de vulnerabilidad social, sus preceptores explicaban que "se pierde un $15 \%$ de alumnos por año en cada curso por inasistencias". Al respecto mostraban la siguiente información correspondiente al mes de agosto: 14 estudiantes (de un total de 30 ) quedaron en condición de libres por inasistencias en primer año, 5 en segundo año de un curso de 37 y 12 en tercer año del Ciclo Básico con 32 alumnos. De los que quedaban, 6 en estos tres cursos habían solicitado la segunda reincorporación por acumulación de inasistencias.

En el año 2011, en otra escuela secundaria de gestión estatal de la capital cordobesa, ubicada en un barrio de raigambre obrera, los preceptores y directivos señalaban su preocupación pues en el mes de abril un primer año con 31 
Revista de la Escuela de Ciencias de la Educación, año 12, nRo. 11, vol. 1, enero a junio de 2016. Páginas 47-68. ISSN 1851-6297. ISSN EN LIINEA 2362-3349. LAS INASISTENCIAS DE LOS ESTUDIANTES: LA ESCUELA SECUNDARIA EN CUESTIÓN. AndREa MARTINo

alumnos/as había acumulado 100 inasistencias; un $3^{\circ}$ año con 30 alumnos/as había acumulado 93 y el $5^{\circ}$ año 107, de un total de 20 días de clases.

En otra institución, también de gestión estatal de la Ciudad de Córdoba, para el año 2013 se registraba el siguiente dato: en un $1^{\circ}$ año del turno tarde con 31 alumnos en el mes de agosto la escuela había contabilizado un total de 412 inasistencias en 20 días de clase. Hacia fines del ciclo lectivo de ese mismo año, un reconocido diario cordobés presentaba tendencias preocupantes sobre el ausentismo. La autora de la nota, una docente, mostraba las siguientes cifras: "Al observar los registros de asistencia de los alumnos, encontramos que un 12 por ciento del total cuya concurrencia ha sido de un 17,3 por ciento del primer trimestre; del 14,28 por ciento del segundo trimestre con promedios en las asignaturas por debajo de dos. En lo que va del tercer trimestre presenció sólo un 7 por cierto de las clases" (La voz del Interior, 10/11/2013). En otra nota con misma fecha, quien ofrece datos es un inspector de una zona del interior provincial. "Los estudiantes faltan muchísimo. Es un problema importante (...) En su zona, diez alumnos de una escuela de 140 estudiantes están en alto riesgo de quedar libres. Un estudiante por ejemplo, faltó 67 veces en el año. “¿Qué proceso de aprendizaje puede hacer ese chico? ¿Cómo sigue Lengua o Matemática?, se preguntó el inspector" (10/11/2013, La Voz del Interior).

Interesa señalar algo en relación a la información presentada. En el segundo y tercer caso, la cifra que la escuela produce se refiere a un dato de la estadística escolar que es la asistencia media. Para quienes no conocen cómo se construye este indicador, se trata de una medida que tiene como unidad el curso, o cada sección de los cursos y no a los estudiantes en particular. Se obtiene a través de la multiplicación entre la cantidad de estudiantes que conforman el curso o sección, la cantidad de inasistencias acumuladas durante un mes por ese grupo, y se lo divide por la cantidad de días de clase. Se trata de una medida que si bien logra reconocer tendencias en la asistencia respecto a cada curso en particular, no puede identificar trayectorias escolares individuales. Ello hace que, por ejemplo, ante el dato del $1^{\circ}$ año con 412 inasistencias acumuladas durante el mes de agosto, resulte imposible conocer quiénes son los estudiantes que han colaborado en la producción de esta cifra y por qué. Lo que aquí se busca señalar es que no alcanzan las formas instituidas de contabilizar las inasistencias de los chicos (4), así como las lecturas que de esta información deben poder derivarse.

Por otra parte, es necesario advertir que si bien el ausentismo de los chicos es una problemática de todos los cursos, las cifras del mismo en los primeros años de la escuela secundaria son alarmantes en relación a sostener algún tipo de continuidad en los aprendizajes. En muchos de estos casos, estamos ante estudiantes que son la primera generación en sus familias en alcanzar este nivel, y que tienen fuertes dificultades en el aprendizaje del oficio de alumno (5), así como en la apropiación de su régimen académico (6). 
Revista de la Escuela de Ciencias de la Educación, año12, nRo.11, vol. 1, enero a Junio de 2016. Páginas 47-68. ISSN 1851-6297. ISSN EN LINEA 2362-3349. LAS INASISTENCIAS DE LOS ESTUDIANTES: LA ESCUELA SECUNDARIA EN CUESTIÓN. AndREa MarTino

\section{Viejas regulaciones para los nuevos modos de ausentismo}

La regulación de la asistencia a clases en la provincia de Córdoba, no ha sido objeto de modificación ni tratamiento. Todavía sigue vigente la Resolución D.E.M.E.S. Nº 979/63, la misma establece en algunos de sus artículos lo siguiente:

"Art. 106: El alumno que durante el curso lectivo incurriera en quince inasistencias, justificadas o no, perderá el curso.

Art. 107: El alumno comprendido en el artículo anterior podrá ser reincorporado siempre que las tres cuartas partes de sus inasistencias sean justificadas. La reincorporación será otorgada por la dirección juntamente con el voto positivo de la mayoría absoluta de los profesores pertenecientes al curso y división al cual concurre el alumno afectado. Los alumnos comprendidos en el artículo anterior seguirán concurriendo a clase y se les computarán las inasistencias, hasta tanto se expida sobre el pedido de reincorporación la dirección del establecimiento. La resolución definitiva deberá producirse en un plazo no mayor de tres días hábiles.

Art. 108: El alumno reincorporado por primera vez que incurriera en diez nuevas inasistencias, justificadas o no, perderá nuevamente su condición de regular, si se tratara de inasistencias justificadas y de un alumno de buena conducta y aplicación, el director juntamente con el voto positivo de la mayoría absoluta de los profesores pertenecientes al curso y división al cual concurre el alumno afectado, podrá otorgarle una segunda reincorporación.

Art. 109: El alumno reincorporado por segunda vez, que incurra en cinco nuevas inasistencias, justificadas o no, pasará a condición de libre. En todos los casos en que este reglamento exige mayorías de profesores para el tratamiento y resolución de reincorporaciones y sanciones deberá computarse en las mismas el voto del preceptor a cargo del curso y división del alumno afectado".

De acuerdo a lo establecido por esta normativa, un estudiante puede acumular 30 inasistencias como máximo a lo largo del ciclo lectivo. Si sobrepasa este límite, pierde su regularidad, y ello significa que debe rendir en calidad de libre todas las asignaturas del curso en que se encuentre inscripto, y si no lo hace, debe repetir.

En los hechos, y tal como se viene poniendo de manifiesto en el trabajo empírico, algunos estudiantes acumulan algo más que treinta inasistencias. Ante estos casos, los preceptores -especialmente pues son la figura institucional a cargo del control de la asistencia de los estudiantes-, a veces en forma solitaria y otras a través de un trabajo colegiado con otras figuras institucionales como los coordinadores de curso, o los propios directivos, estiran hasta donde pueden el sostenimiento de la condición de alumno regular. Es esto lo que se pierde 
Revista de la Escuela de Ciencias de la Educación, año12, nRo.11, vol. 1, enero a Junio de 2016. Páginas 47-68. ISSN 1851-6297. ISSN EN LIINEA 2362-3349. LAS INASISTENCIAS DE LOS ESTUDIANTES: LA ESCUELA SECUNDARIA EN CUESTIÓN. AndREa Martino

cuando un alumno falta de manera excesiva a la escuela, según lo establece la regulación vigente. Resulta interesante remarcar esto, pues en general, lo que aparece en un sentido común escolar bastante difundido tanto entre docentes como entre estudiantes, es que cuando un chico/a queda libre por faltas, queda fuera de la escuela. Sigue siendo alumno de ella, sin embargo lo que perdió es su condición de regularidad.

Otra cuestión que interesa remarcar alude a lo que se considera inasistencia. De acuerdo a la normativa vigente, se señala lo siguiente:

Art. 101: Considérase inasistencia:

a) La falta de concurrencia a la escuela en día de actividad escolar o a los actos oficiales en que éste intervenga

b) La ausencia del alumno sin autorización, en cualquiera de las horas de clase, aún cuando haya concurrido a las restantes, sin perjuicio de la sanción correspondiente.

De acuerdo a ello, la asistencia regular a la escuela secundaria se contabiliza por jornada escolar y no por espacio curricular, tal como sí ocurre para la modalidad de adultos. Nuevamente aquí interesa interrogarse sobre un formato escolar y una temporalidad monocrónica que no logra contemplar condiciones subjetivas y sociales y proponer alternativas más flexibles para que los chicos puedan permanecer y aprender en la escuela.

\section{Condiciones y obstáculos en la presencia de los estudiantes...}

En el trabajo de campo realizado hasta el momento (7) las llegadas tarde y las faltas en educación física suelen ser una de las principales productoras de inasistencias según lo que cuentan los propios estudiantes. Del total de entrevistados todos han repetido alguna vez, en primaria o en secundaria, o en ambos niveles. En este último caso, las repitencias se derivaban del ausentismo excesivo.

Se trataría, en este caso de dos modos de ausencia. Una ligada a las formas instituidas de distribución de las asignaturas escolares a lo largo de la jornada. Tal es el caso de educación física que se dicta en contraturno y que suele desordenar cotidianos familiares inestables en su resoluciones organizativas, puesto que en la mayoría de los jóvenes entrevistados, el horario de entrada a la escuela era a las $13.30 \mathrm{hs}$, mientras que educación física se dictaba en el horario de 8.30 a 9.30 hs. de la mañana. Estos jóvenes, en su mayoría provenientes de pueblos aledaños a donde está ubicada la escuela, debían tomar varios colectivos para asistir a estos dos horarios.

Entrevistadora: Vos estás con 27 faltas... ¿por qué tantas?

Estudiante: Porque la mayoría... por gimnasia...

Entrevistadora: ¿No venís a gimnasia? 
Revista de la Escuela de Ciencias de la Educación, año12, nRo.11, vol. 1, enero a Junio de 2016. Páginas 47-68. ISSN 1851-6297. ISSN EN LINEA 2362-3349. LAS INASISTENCIAS DE LOS ESTUDIANTES: LA ESCUELA SECUNDARIA EN CUESTIÓN. AndREa MarTino

Estudiante: No... porque si venía, pero los lunes como que no puedo... porque juego al fútbol los domingos y me levanto los domingos, entonces los lunes no voy... así puedo ir a clase bien...

Entrevistadora: ¿Y a qué hora tienen gimnasia?

Estudiante: De ocho y media a nueve y media.

Entrevistadora: Pero vos venís a la tarde, los lunes supuestamente vos tenías de ocho y media a nueve y media y después te volvías a tu casa y volvías a la escuela para entrar a clase?

Estudiante: Claro, me tomaba el de las doce... para llegar.

Entrevistadora: O sea que vos ibas y venías, ibas y venías...

Estudiante: Sí, y a veces no me daba ganas... a la mañana estoy solo, mi mamá no está (porque trabaja) y mi hermana siempre está con el novio... (su padre no vive con ellos actualmente).

Otra forma de ausencia que fueron planteando los estudiantes se encuentra articulada a la forma de concebir la jornada escolar en la educación secundaria como una unidad indivisible y compacta que no puede desarmarse para ser más flexible frente a ciertas circunstancias vitales. Tal es el caso de las llegadas tarde, debido a horarios y organizaciones familiares que no suelen acompañar en muchos casos las normativas escolares. Escuchemos lo que nos dicen algunos jóvenes:

Estudiante 1: Falté no más cinco o seis veces. Llego tarde porque a veces salgo muy tarde, porque me levanto tarde y hasta que me baño y como, y a veces el colectivo se demora mucho... Vos tratás de explicarlo y te ponen ausente por llegar tarde igual... es al pedo no más porque tenés la falta...

Estudiante 2: Tengo muchas faltas por las llegadas tarde... no falto mucho... llego tarde porque mi mamá se pone a cocinar tarde, y hasta que como, se me pasa el horario...

Estudiante 3: Por el colectivo así, se me iba el colectivo de las doce y media y venía otro y era así... y tenía problemas en mi casa de comidas... cuando tenía que comer, en el horario en que tenía que comer no estaba la comida, recién la estaban haciendo.

También son muy numerosos los casos de adolescentes que se ausentan de la escuela por asumir responsabilidades en la organización de sus hogares, como el cuidado de los hermanos ya sea por cuestiones laborales de los padres, o por alguna enfermedad. Si bien en este trabajo no se abordará con exhaustividad esta cuestión, sí es necesario señalar que esta es una de las principales razones, la más extendida en el grupo de estudiantes entrevistados (casi el $80 \%$ del total). Se trata de jóvenes que en general son del grupo de los hijos mayores de familias que logran acceder a este nivel por primera vez. Muchos de estos estudiantes tienen sus hermanos mayores estudiando en ins- 
Revista de la Escuela de Ciencias de la Educación, año12, nRo.11, vol. 1, enero a Junio de 2016. Páginas 47-68. ISSN 1851-6297. ISSN EN LIINEA 2362-3349. LAS INASISTENCIAS DE LOS ESTUDIANTES: LA ESCUELA SECUNDARIA EN CUESTIÓN. AndREa Martino

tituciones para adultos, porque han fracasado en la escuela secundaria común por las mismas razones que en la actualidad a ellos los sitúan en situaciones límites para permanecer.

Estudiante: Tengo un par de problemas en casa, están separados mis papás y hay veces que me tengo que quedar a cuidar a mi mamá. Somos cinco hermanos...

Entrevistadora: ¿Vos sos el mayor?

Estudiante: No, el del medio. Somos tres varones y dos mujeres...

Entrevistadora: Y dos de ustedes vienen a la escuela secundaria...?

Estudiante: $Y$ dos al $F$ (se refiere al nombre de la escuela primaria).

(...) Para mí es más importante la escuela, a veces yo quiero venir, pero no puedo... eso es lo que le quería decir, yo a veces quiero venir pero no puedo.

Entrevistadora: ¿Tu mamá te pide que la ayudes?

Estudiante: No, no... ella siempre me dice que venga, pero yo veo que sola no puede... porque a veces no puede... vio como son las mujeres, te dicen no pero es sí... yo le pregunto ¿estás bien?, ¿estás bien?, sí, me dice ella y yo sé que no está bien... ella me dice está todo bien J, anda, y yo sé que no está todo bien y necesita una ayuda. Estudiante: Sí, a dos cuadras vive mi hermana... ella está haciendo el terreno y se sabe venir, algunas veces ella encargaba el material, y tenía que subirlo con el marido, entonces yo me quedaba a cuidar a mis sobrinos, pero no era siempre.

En algunos casos, nos encontramos con relatos signados por la poca previsión propia y familiar en el uso de las inasistencias durante el año. Esto implica que algunos estudiantes las acumularon en los primeros meses del año, y luego situaciones familiares en especial enfermedades, los obligaron a ausentarse de la escuela, pero sin márgenes para más reincorporaciones.

Estos registros dan cuenta de una forma escolar que todavía sigue siendo poco flexible ante ciertas condiciones y cotidianos de los chico/as, de los cuales no son responsables exclusivamente, pero al fin y al cabo sobre ellos recae la "falta". En estos casos, no se trataría de ausencias prolongadas, sino de una acumulación sostenida a lo largo del año de ausencias, que se torna crítica en el último trimestre del ciclo lectivo. En este sentido, interesa volver a recuperar el planteo de Terigi sobre los modos de ausentismo, para reconocer su reverso, esto es "modos de estar presente en la escuela". No es lo mismo un joven que está presente en la escuela aunque llegó tarde, que aquel que faltó a toda la jornada escolar, o de quien falta solo a educación física, o de aquellos que se ausentan un mes entero, y en este último caso, no es lo mismo aquel que falta en los últimos meses del año lectivo porque se incorpora a trabajar en los circuitos turísticos, que aquellos estudiantes que se ausentan en mayo o 
Revista de la Escuela de Ciencias de la Educación, año12, nRo.11, vol. 1, enero a Junio de 2016. Páginas 47-68. ISSN 1851-6297. ISSN EN LIINEA 2362-3349. LAS INASISTENCIAS DE LOS ESTUDIANTES: LA ESCUELA SECUNDARIA EN CUESTIÓN. AndREa MarTino

en agosto. Estaríamos hablando entonces de una presencialidad múltiple que necesita ser reconocida pedagógicamente para pensar estrategias diferentes según el caso.

Entre estas formas de ausencias, interesa señalar otra que, a diferencia de las anteriores, no estaría directamente relacionada con condiciones sociales de existencia y con cotidianos domésticos mayor o menormente organizados en torno a lo escolar. Lo que va apareciendo también en los relatos de los estudiantes entrevistados tiene que ver con "ausencias decididas", es decir, hay una decisión personal de no asistir ese día a clases. En muchos de estos casos, dichas decisiones comienzan a ser reiteradas, por lo menos dos a la semana durante un tiempo prolongado, lo cual sitúa a los jóvenes en el límite de perder la regularidad, quedar libres y por tanto rendir todas las asignaturas o bien repetir de año. Escuchemos sus voces y la de su preceptora al referirse a este tipo de inasistencias:

Estudiante: ...el año pasado fue un capricho de no hacer nada... Me quedaba en mi casa. No me daban muchas ganas de venir, aunque mi mamá me decía, tengo que venir de allá (se refiere a un pueblo aledaño a la localidad en la que se encuentra la escuela) y me daba fiaca...

Entrevistador: ¿Y por qué te quedaste libre en primero?

Estudiante: Lo mismo que ahora, me colgué con las faltas y me quedé libre...

Entrevistador: ¿Cómo es eso de te colgás?

Estudiante: (se ríe) No sé, falté... no llevé la cuenta... sí veo y digo «uy no falto más y falto〉...

Estudiante: Sí, pasé a segundo... cambié una banda... porque era un desastre...

Entrevistador: ¿Cómo es eso? ¿En primero por qué repetiste?

Estudiante: Por las faltas, me llevaba dos no más (se refiere a las materias), y me quedé por las faltas...

Entrevistador: ¿Tenías muchas faltas?

Estudiante: Como 42. Me quedé libre (...) ahora entendí un poco que no sirve de nada eso. De a poco fui entendiendo...

Preceptora: Por lo general coincide con alumnos que tienen muchas dificultades, que en realidad no tienen como muy en claro qué lugar debiera ocupar la escuela en su vida... en algunos casos juegan con el límite... y ellos como que no registran y creen que ellos pueden hacer siempre lo que desean, que las normas están para otros... Se quedan en la calle, en el puente, tomando una coca, y te dicen después 'no pintaba entrar a la escuela'... 
Revista de la Escuela de Ciencias de la Educación, año 12, nRo. 11, vol. 1, enero a junio de 2016. Páginas 47-68. ISSN 1851-6297. ISSN EN LIINEA 2362-3349. LAS INASISTENCIAS DE LOS ESTUDIANTES: LA ESCUELA SECUNDARIA EN CUESTIÓN. AndREa MARTINo

La extensión del tiempo escolar para permanecer a lo largo de toda la escuela secundaria, por la vía de la obligatoriedad, no transforma por sí misma el conjunto de condiciones sociales en las que se construyen las prácticas de escolarización de los estudiantes y sus familias, en especial de aquellos que guardan mayores distancias culturales respecto de los códigos de lo escolar y que en general se encuentran en situaciones de vulnerabilidad social.

Parte de los desafíos que hoy se juegan en la construcción social de la obligatoriedad, y tal como venimos viendo, se juegan en que aquellos estudiantes de la escuela secundaria que antes no ingresaban a ella, y hoy sí lo hacen, puedan "convertirse en sus estudiantes de un modo sostenido" y puedan egresar de este nivel.

En el relato de los jóvenes, hemos visto la complejidad de las articulaciones entre la escuela secundaria, con sus normativas y exigencias, y la reproducción social de sus familias. Incorporar la secundaria como parte de la vida de los adolescentes y de los horizontes familiares, implica inversiones de sentido, tiempo, organización, dinero, sostenidas durante un plazo de varios años, no necesariamente disponibles en algunas de las familias de los adolescentes más pobres, menos aún cuando algo en el orden de la resolución de la subsistencia está en juego, y reclama el aporte de los hijos en el sostén de la vida doméstica.

La normativa sobre el régimen académico, particularmente la exigencia de presencialidad, opera sobre condiciones sociales y familiares desiguales; condiciones en las que la experiencia social de la escolaridad secundaria, para los estudiantes más pobres y desamparados, se vuelve una rutina difícil de construir y sostener, tanto en el tiempo como en su significatividad.

\section{Temporalidades en la experiencia escolar de algunos adolescentes...}

Ser alumno de una escuela (en este caso secundaria) introduce a los jóvenes en una experiencia temporal en la que se configuran distinciones entre tiempo escolar y tiempo libre, tiempo de clases y tiempo de vacaciones, horas de clase, recreos, horas libres. Distinciones que se distribuyen en la jornada diaria, semanal y anual, según el caso, de acuerdo a las normativas del sistema educativo y específicas para cada nivel.

Traemos aquí la situación de dos estudiantes cuya ausencia extendida interpela la temporalidad del sistema educativo. El año lectivo de estos estudiantes parece estar jalonado por etapas donde no se asiste a la escuela, no se sabe muy bien por qué, las razones predominantes son el no tener ganas y por momentos donde ciertas circunstancias familiares y/o domésticas obligan a ausentarse, y a seguir acumulando inasistencias.

Milena asistió solo cuatro veces en setiembre... en octubre faltó todo el mes, y en noviembre comenzó a venir bien... relataba su preceptora de $2^{\circ}$ año. 
Revista de la Escuela de Ciencias de la Educación, año12, nRo.11, vol. 1, enero a Junio de 2016. Páginas 47-68. ISSN 1851-6297. ISSN EN LIINEA 2362-3349. LAS INASISTENCIAS DE LOS ESTUDIANTES: LA ESCUELA SECUNDARIA EN CUESTIÓN. AndREa Martino

Al momento de las entrevistas, no tiene promedio en ninguna asignatura. Frente a la pregunta por tantas inasistencias, señala que ello se debió a la enfermedad de su madre.

Rocío tiene 14 años y está en segundo año del ciclo básico. Tiene una excesiva cantidad de inasistencias. De hecho en el mes de abril del 2014 asistió una semana y media a clases. Señala que simplemente falta, que le dice a su padre que ese día no hay clases y que éste le cree o simplemente permite que falte, aún cuando pasa toda una semana sin asistir a la escuela. Pese a sus reiteradas inasistencias, piensa terminar segundo año, levantar los promedios y pedir el pase a otra escuela.

En estos casos nos encontramos con adolescentes que durante un mes o casi dos faltan a la escuela, se desenganchan un tiempo, y luego vuelven. En las entrevistas realizadas con ellas (y con otros) lo que sorprendía es que frente a la propia percepción (la del investigador) de una temporalidad sobre lo escolar fragmentada que seguramente producía efectos en los sentidos sobre la escuela y el aprendizaje, estos jóvenes no significaban su ausencia prolongada como un desenganche o una fragmentación, sino más bien como una interrupción transitoria, de la cual, una vez superadas ciertas dificultades, les permitía regresar a la escuela. Veamos el siguiente fragmento de entrevista:

Entrevistador: Faltar un mes y medio continuado es como mucho, me parece a mí.

Estudiante: Sí, pero después vuelvo y termino...

Entrevistador: Luego de todo ese tiempo ¿pensaste en dejar la escuela?

Estudiante: No, ni a palos... pero no sabía si en la escuela me iban a recibir de nuevo... Pero en la escuela me recibieron de nuevo, por suerte... yo la quiero terminar.

Estudiante: Sí... la otra vez falté mucho... no venía... pero ahora volví y estoy levantando las materias... mi hermana me está ayudando... y tengo una prima que es maestra particular...

Entrevistador: ¡Qué bueno!, ¿este año venís faltando bastante no? Me dijo tu preceptora que casi un mes entero estuviste sin venir...

Estudiante: Sí... y el año pasado repetí también por las faltas...

Entrevistador: ¿Y qué pasa que dejás de venir?

Estudiante: Es que... el año pasado mi mamá se enfermó mucho y mi papá la cuidaba cuando estaba internada... ¿cómo le explico?... Uno a veces no puede... tiene que dejar porque hay otras cosas y después vuelvo...

En sus relatos y en el de otros estudiantes, la interrupción en la asistencia a clase no es sinónimo de discontinuidad en su proceso escolar. Los sentidos 
Revista de la Escuela de Ciencias de la Educación, año 12, nRo. 11, vol. 1, enero a junio de 2016. Páginas 47-68. ISSN 1851-6297. ISSN EN LIINEA 2362-3349. LAS INASISTENCIAS DE LOS ESTUDIANTES: LA ESCUELA SECUNDARIA EN CUESTIÓN. ANDREA MARTINO

que construyen sobre el mismo parecerían articularse a una experiencia vital y temporal que aparece más ligada a la idea de intermitencia que de continuidad y por tanto no coincide con la temporalidad regular que establece el sistema educativo. Es en esta dirección que interesa formular la pregunta acerca de cómo lo escolar, uno de cuyos rasgos organizativos es el de la regularidad de la presencia, se va produciendo en el seno de una presencialidad irregular.

Ante la pregunta por sus aprendizajes, estos estudiantes señalan que completan la carpeta, piden que algún compañero o familiar explique los temas nuevos, y que con eso alcanza. En ninguno de los entrevistados se percibió algo en el orden de la discontinuidad, y del quedar ajeno al desarrollo de las clases. Respecto a las evaluaciones señalan la existencia de nuevas oportunidades por parte de los profesores. Tampoco lograron dar mayores precisiones acerca de las estrategias pedagógicas implementadas por sus profesores para abordar su ausencia extendida.

Interesa señalar que en todos estos casos, el conocimiento anticipado o probable de que hay chances académicas para aprobar es el criterio que sostiene el esfuerzo por volver a ponerse al día, es decir solo cuando se sabe que es posible aprobar la materia porque se tiene promedio, o es posible obtenerlo, se continúa. En los casos contrarios se trata de estudiantes que abandonan sin más.

\section{Consideraciones finales}

Una pregunta que se viene sosteniendo en este último tiempo, es que si la presencialidad es condición para el cumplimiento de derechos educativos en la escuela, cuando la misma se quiebra ante la excesiva inasistencia de los/as estudiantes, ¿de qué manera es posible efectivizarlos desde el actual formato escolar? Interesa sostener esta pregunta, desde el supuesto de que cuando lo/as chico/as no están en la escuela, por las razones que fuera, la apuesta de inclusión escolar se resiente.

La ausencia de los estudiantes en las aulas se constituye, en este sentido, en un analizador de las tensiones entre las políticas públicas de las últimas décadas, tendientes a reconocer derechos (en este caso educativos) y los posicionamientos de los sujetos frente a ellos, tanto de los docentes/directivos para hacerlos cumplir como de los propios estudiantes para constituirse en sus beneficiarios. Las inasistencias escolares de los estudiantes, desde la presencialidad, inhabilitan tanto a unos como a otros para su cumplimiento y obligan a pensar en otras formas -aunque sea provisorias- para que los chicos sigan siendo alumnos de una escuela.

Las inasistencias de los estudiantes a la escuela secundaria se constituyen en un problema cuando su acumulación excesiva torna discontinuo, interrumpe y disminuye su tiempo de aprendizaje. Creemos que situarnos en esta pers- 
Revista de la Escuela de Ciencias de la Educación, año12, nRo.11, vol. 1, enero a Junio de 2016. Páginas 47-68. ISSN 1851-6297. ISSN EN LIINEA 2362-3349. LAS INASISTENCIAS DE LOS ESTUDIANTES: LA ESCUELA SECUNDARIA EN CUESTIÓN. AndREa MARTINo

pectiva, sin reducir la mirada a la contabilización de "faltas" acumuladas, nos permite reconocerlo como un problema pedagógico e institucional, pero a la vez, también, nos ha permitido indagar en los sentidos y experiencias que los jóvenes y adolescentes van construyendo y sosteniendo sobre su tiempo de escolaridad, mientras ellos mismos transitan y atraviesan otros tiempos vitales. En esta línea nos ha interesado mirar el modo en cómo los tiempos de los estudiantes y los sentidos que se van jugando en su duración y uso tensionan el principio de democratización del tiempo escolar promovido por las políticas educativas de la última década, al tener entre sus medidas la ampliación del tiempo escolar, entendido éste como un tiempo de derechos.

Gimeno Sacristán distingue entre tiempo escolar y tiempo educativo, señalando que los estudiantes aprenden otras cosas, en otros espacios y momentos de su vida además del destinado a la escuela. Escribe "el tiempo de la escolarización no es el de toda la educación” (Gimeno Sacristán, 2008).

Interesa recuperar esta distinción para preguntarnos: ¿Qué sucede cuando el tiempo escolar comienza a agujerearse, a llenarse de vacíos y se fragmenta a través del ausentismo? ¿Qué sucede cuando esto ocurre y no existen otras formas complementarias de tiempo educativo que le posibiliten a los niños y jóvenes inscribirse en las tramas de una sociedad como ciudadanos de pleno derecho, accediendo a los bienes culturales que ha construido la humanidad? ¿Es posible pensar formas de continuidad y regularidad en los aprendizajes de lo/as estudiantes aún en situaciones de irregularidad de su asistencia a clases?

Si los jóvenes de los sectores sociales más desfavorecidos no asisten a la escuela, pero tampoco a otro tipo de espacios socio educativos ¿Cómo logran encontrarse y apropiarse de los bienes y los códigos de ciudadanía que les permitan en sociedades como la nuestras ser miembros plenos en ellas? En este sentido se considera que el lugar del Estado es central para el desarrollo de políticas sociales y culturales que en forma complementaria a lo que hacen las escuelas, generen mejores condiciones para la asistencia constante de los jóvenes a ella.

\section{Notas Bibliográficas}

(1) Este artículo recupera algunos planteos y desarrollos realizados en el trabajo presentado en el VI Coloquio Internacional del IIFAP "Estado, política pública y acción colectiva: Praxis emergentes y debates necesarios en la construcción de la democracia", realizado durante los días $1^{\circ}$ y 2 de Octubre de 2015, Córdoba, Argentina; y del trabajo presentado en el III Congreso Internacional de Educación. "Formación, sujetos y prácticas", abril de 2016, Universidad Nacional de La Pampa.

(2) La Fundación SES es una organización social que se dedica a la promoción y al desarrollo de diferentes estrategias para la inclusión de los adolescentes y jóvenes con menos oportunidades trabajando desde la perspectiva de los derechos de los jóvenes. Al respecto véase: http://www.fundses.org.ar 
Revista de la Escuela de Ciencias de la Educación, año12, nRo.11, vol. 1, enero a Junio de 2016. Páginas 47-68. ISSN 1851-6297. ISSN EN LIINEA 2362-3349. LAS INASISTENCIAS DE LOS ESTUDIANTES: LA ESCUELA SECUNDARIA EN CUESTIÓN. AndREa Martino

(3) La información que se presenta fue obtenida en el marco de prácticas e intervenciones profesionales de asesoramiento y acompañamiento en estas instituciones escolares. A la hora de presentar este trabajo, se solicitó permiso para publicar estos datos a los directivos de las mismas.

(4) La asistencia media de los estudiantes en la escuela secundaria no es un dato que aparece en la estadística de la DINIECE, ni tampoco en las estadísticas oficiales de la provincia de Córdoba.

(5) Perrenoud afirma que el triunfo en la escuela requiere de la construcción del oficio de alumno. Ello involucra cuestiones muy diversas como el aprendizaje del currículum oculto, de estrategias en las evaluaciones para obtener buenos resultados. En la escuela se aprende la disciplina, los buenos hábitos, la conformidad intelectual, el sentido común, el compromiso con el trabajo, el afán de excelencia y la habilidad táctica ante las evaluaciones (2009 $4^{\circ}$ ed.).

(6) Se entiende el régimen académico al conjunto de regulaciones sobre la organización de las actividades de los alumnos y sobre las exigencias a las que éstos deben responder (Camilloni, 1991).

(7) Hasta el momento, se ha entrevistado a estudiantes de $2^{\circ}$ y $4^{\circ}$ año. La elección de estos dos cursos, se debió en parte a la disponibilidad de los mismos para llevar adelante entrevistas; también porque se buscaba dos cursos representativos de cada ciclo. Los preceptores ofrecieron información sobre un grupo de estudiantes en estos cursos que en los momentos de las entrevistas acumulaban inasistencias y se encontraban en peligro de quedar libres. No se pudo contactar con estudiantes que durante el año habían quedado libres y ya no asistían a la escuela.

\section{Referencias Bibliográficas}

- Binstock G. y Cerrutti M. (2005). Carreras truncadas. El abandono escolar en el nivel medio en la Argentina. Buenos Aires: Unicef.

- Camilloni A. (1991). Alternativas para el régimen académico. Revista Iglú, № 1. Buenos Aires.

- DINIECE-Unicef, (2004). Las dificultades en las trayectorias escolares de los alumnos. Un estudio en escuelas de nuestro país. Ministerio de Educación, Ciencia y Tecnología de Argentina y Dirección Nacional de Información y Evaluación de la Calidad Educativa.

- Gaminián A. (2014). Pistas para mejorar. ¿Qué hicieron los países, escuelas y estudiantes con mejor desempeño en el Programa para la Evaluación Internacional de Alumnos (PISA) 2012? Buenos Aires, Argentina. Proyecto Educar 2050. Asociación Civil para la mejora de la educación en Argentina.

- Gimeno Sacristán J. (2008). El valor del tiempo en educación. Madrid: Editorial Morata.

- Jacinto C. y Freytes Frey A. (2004). Políticas y estrategias para el mejoramiento de las oportunidades de los jóvenes Estudio en la Ciudad de Buenos Aires. Buenos Aires. UNESCO: Instituto Internacional de Planeamiento de la Educación.

- Jacinto C. y Terigi F. (2007). ¿Qué hacer ante las desigualdades en la educación secundaria? Aportes de la experiencia latinoamericana. Buenos Aires: Editorial Santillana.

- Perrenoud P. (2009 $4^{\circ}$ edición). La construcción del éxito y del fracaso escolar. Hacia un análisis del éxito, del fracaso y de las desigualdades como realidades construidas por el sistema escolar, Madrid: Editorial Morata.

- SITTEAL-OEI-UNESCO-Instituto Internacional de Planeamiento de la Educación, (2008). La escuela y los adolescentes. Informe sobre tendencias sociales y educativas 
Revista de la Escuela de Ciencias de la Educación, año12, nRo.11, vol. 1, enero a Junio de 2016. Páginas 47-68. ISSN 1851-6297. ISSN EN LINEA 2362-3349. LAS INASISTENCIAS DE LOS ESTUDIANTES: LA ESCUELA SECUNDARIA EN CUESTIÓN. AndREa MARTIno

en América Latina. IIPE - UNESCO, Sede Regional Buenos Aires. OEI Organización de Estados Iberoamericanos para la Educación, la Ciencia y la Cultura, Madrid.

- Terigi, F. (2007). Los desafíos que plantean las trayectorias escolares. III Foro Latinoamericano de Educación. Jóvenes y docentes. La escuela secundaria en el mundo de hoy.

- (2010). Las cronologías de aprendizaje: un concepto para pensar las trayectorias escolares. Jornada de apertura Ciclo Lectivo 2010, Santa Rosa, La Pampa.

(2010). Las trayectorias escolares como objeto de las políticas públicas. Desafíos y perspectivas. En Primeras Jornadas de Intercambio federal: Experiencias de reorganización de las trayectorias escolares en el nivel primario. Tercera Mesa Federal de Directores.

- Viñar M. (2013). Mundos adolescentes y vértigo civilizatorio. Buenos Aires: Editorial Noveduc. 